\title{
Improvised Nuclear Device
}

National Cancer Institute

\section{Source}

National Cancer Institute. Improvised Nuclear Device. NCI Thesaurus. Code C120576.

A crude nuclear device built from the components of a stolen weapon or from scratch using nuclear material (plutonium or highly enriched uranium). 